\title{
On the fourth power mean of the two-term exponential sums
}

Minhui Zhu' and Di Han ${ }^{2 *}$

A retraction article was published for this article. It is available from the following link: http://www.journalofinequalitiesandapplications.com/content/2014/1/344.

\section{"Correspondence:}

handi515@163.com

${ }^{2}$ Department of Mathematics,

Northwest University, Xi'an, Shaanxi,

P.R. China

Full list of author information is

available at the end of the article

\begin{abstract}
The main purpose of this paper is using the analytic methods and the properties of Gauss sums to study the computational problem of one kind of fourth power mean of two-term exponential sums, and to give an interesting identity and asymptotic formula for it.

MSC: $11 \mathrm{~L} 05$

Keywords: the two-term exponential sums; fourth power mean; Gauss sums; identity; asymptotic formula
\end{abstract}

\section{Introduction}

Let $q \geq 3$ be a positive integer. For any integers $m$ and $n$, the two-term exponential sum $C(m, n, k ; q)$ is defined as follows:

$$
C(m, n, k ; q)=\sum_{a=1}^{q} e\left(\frac{m a^{k}+n a}{q}\right),
$$

where $e(y)=e^{2 \pi i y}$.

About the various properties of $C(m, n, k ; q)$, some authors had studied it, and they obtained a series of results, some related works can be found in references [1-5]. For example, Gauss' classical work proved the remarkable formula (see [6])

$$
C(1,0,2 ; q)=\frac{1}{2} \sqrt{q}(1+i)(1+e(-q / 4))= \begin{cases}\sqrt{q}, & \text { if } q \equiv 1 \bmod 4 \\ 0, & \text { if } q \equiv 2 \bmod 4 \\ i \sqrt{q}, & \text { if } q \equiv 3 \bmod 4 \\ (1+i) \sqrt{q}, & \text { if } q \equiv 0 \bmod 4\end{cases}
$$

where $i^{2}=-1$.

Generally, for any odd number $q$ and $(2 m, q)=1$, the exact value of $|C(m, n, 2 ; q)|$ is $\sqrt{q}$ (e.g. see Berndt, Evans, Williams and Apostol's related works). Cochrane and Zheng [2] show for the general sum that

$$
|C(m, n, k ; q)| \leq k^{\omega(q)} q^{\frac{1}{2}},
$$

where $\omega(q)$ denotes the number of all distinct prime divisors of $q$. 
In this paper, we study the fourth power mean of the two-term exponential sum $C(m, n, k ; q)$ as follows:

$$
\sum_{m=1}^{q}|C(m, n, k ; q)|^{4}
$$

where $n$ is any integer with $(n, q)=1$.

As regards this problem, it seems that none has yet studied it, at least we have not seen any related result before. The problem is interesting, because it can reflect that the mean value of $C(m, n, k ; q)$ is well behaved. The main purpose of this paper is to show this point. That is, we shall prove the following conclusion.

Theorem Let $p>3$ be a prime. Then for any integer $n$ with $(n, p)=1$, we have the identity

$$
\begin{aligned}
& \sum_{m=1}^{p}\left|\sum_{a=1}^{p-1} e\left(\frac{m a^{3}+n a}{p}\right)\right|^{4} \\
& \quad= \begin{cases}2 p^{3}-3 p^{2}-3 p, & \text { if } 3 \nmid p-1, \\
2 p^{3}-5 p^{2}-15 p+4 \tau^{3}\left(\chi_{1}\right)+4 \tau^{3}\left(\bar{\chi}_{1}\right), & \text { if } 3 \mid p-1,\end{cases}
\end{aligned}
$$

where $\chi_{1}$ is any 3-order character mod $p$.

Note that for any non-principal character $\chi \bmod p$, we have $|\tau(\chi)|=\sqrt{p}$, so from our theorem we may immediately deduce the following.

Corollary Let $p>3$ be a prime with $3 \mid p-1$. Then for any integer $n$ with $(n, p)=1$, we have the asymptotic formula

$$
\sum_{m=1}^{p}\left|\sum_{a=1}^{p-1} e\left(\frac{m a^{3}+n a}{p}\right)\right|^{4}=2 p^{3}-5 p^{2}+O\left(p^{\frac{3}{2}}\right) .
$$

It seems that our method can also be used to deal with (1.1) for all prime $p$ and integer $k \geq 4$. But this time, the computing is very complex.

For any integer $h \geq 3$, whether there exists an exact computational formula for

$$
\sum_{m=1}^{p}\left|\sum_{a=1}^{p-1} e\left(\frac{m a^{3}+n a}{p}\right)\right|^{2 h}
$$

where $p$ is an odd prime and $(n, p)=1$, is an open problem.

\section{Several lemmas}

In this section, we will give several lemmas which are necessary in the proof of our theorem. In the proving process of all lemmas, we used many properties of Gauss sums; all these can be found in [6], we will not be repeat them here. First we have the following. 
Lemma 1 Let $p$ be an odd prime, $\chi$ be any non-principal character mod $p$. Then for any integer $n$ with $(n, p)=1$, we have the identity

$$
\begin{aligned}
& \left.\left|\sum_{m=1}^{p-1} \chi(m)\right| \sum_{a=1}^{p-1} e\left(\frac{m a^{3}+n a}{p}\right)\right|^{2} \mid \\
& \quad= \begin{cases}p\left|\sum_{a=1}^{p-2} \bar{\chi}\left(3 a^{2}+3 a+1\right)\right|, & \text { if } \chi \text { is not a 3-order character mod } p, \\
\sqrt{p}\left|-2+\sum_{a=1}^{p-1} \bar{\chi}(a(1-a))\right|, & \text { if } \chi \text { is a } 3 \text {-order character mod } p .\end{cases}
\end{aligned}
$$

Proof Note that $\chi$ is a non-principal character $\bmod p$, so if $\chi$ is not a 3-order character $\bmod p$ (that is, $\chi^{3} \neq \chi_{0}$, the principal character $\bmod p$ ), then from the properties of Gauss sums we have

$$
\begin{aligned}
& \sum_{m=1}^{p-1} \chi(m)\left|\sum_{a=1}^{p-1} e\left(\frac{m a^{3}+n a}{p}\right)\right|^{2} \\
& =\sum_{a=1}^{p-1} \sum_{b=1}^{p-1} \sum_{m=1}^{p-1} \chi(m) e\left(\frac{m\left(a^{3}-b^{3}\right)+n(a-b)}{p}\right) \\
& =\sum_{a=1}^{p-1} \sum_{b=1}^{p-1} \sum_{m=1}^{p-1} \chi(m) e\left(\frac{m b^{3}\left(a^{3}-1\right)+n b(a-1)}{p}\right) \\
& =\tau(\chi) \sum_{a=1}^{p-1} \bar{\chi}\left(a^{3}-1\right) \sum_{b=1}^{p-1} \bar{\chi}^{3}(b) e\left(\frac{n b(a-1)}{p}\right) \\
& =\tau(\chi) \tau\left(\bar{\chi}^{3}\right) \sum_{a=1}^{p-1} \bar{\chi}\left(a^{3}-1\right) \chi^{3}(n(a-1)) \\
& =\chi^{3}(n) \tau(\chi) \tau\left(\bar{\chi}^{3}\right) \sum_{a=1}^{p-2} \bar{\chi}\left((a+1)^{3}-1\right) \chi\left(a^{3}\right) \\
& =\chi^{3}(n) \tau(\chi) \tau\left(\bar{\chi}^{3}\right) \sum_{a=1}^{p-2} \bar{\chi}\left((\bar{a}+1)^{3}-\bar{a}^{3}\right) \\
& =\chi^{3}(n) \tau(\chi) \tau\left(\bar{\chi}^{3}\right) \sum_{a=1}^{p-2} \bar{\chi}\left(3 a^{2}+3 a+1\right),
\end{aligned}
$$

where $\tau(\chi)=\sum_{a=1}^{p-1} \chi(a) e\left(\frac{a}{p}\right)$ denotes the classical Gauss sum.

If $\chi$ is a 3 -order character mod $p$, then $\chi^{3}=\chi_{0}$; note that for any integer $a$ with $(a, p)=1$, we have

$$
\chi^{2}(a)+\chi(a)+1= \begin{cases}3, & \text { if } a \text { is a third residue } \bmod p, \\ 0, & \text { otherwise. }\end{cases}
$$

From the method of proving (2.1) we have the identity

$$
\begin{aligned}
& \sum_{m=1}^{p-1} \chi(m)\left|\sum_{a=1}^{p-1} e\left(\frac{m a^{3}+n a}{p}\right)\right|^{2} \\
& \quad=\tau(\chi) \sum_{a=1}^{p-1} \bar{\chi}\left(a^{3}-1\right) \sum_{b=1}^{p-1} \bar{\chi}^{3}(b) e\left(\frac{n b(a-1)}{p}\right)
\end{aligned}
$$




$$
\begin{aligned}
& =\tau(\chi) \sum_{a=1}^{p-1} \bar{\chi}\left(a^{3}-1\right) \sum_{b=1}^{p-1} e\left(\frac{n b(a-1)}{p}\right)=-\tau(\chi) \sum_{a=1}^{p-1} \bar{\chi}\left(a^{3}-1\right) \\
& =-\tau(\chi) \sum_{a=1}^{p-1}\left(\chi^{2}(a)+\chi(a)+1\right) \bar{\chi}(a-1) \\
& =-\tau(\chi)\left(\sum_{a=1}^{p-1} \bar{\chi}(a-1)+\sum_{a=1}^{p-1} \bar{\chi}(\bar{a}(1-\bar{a}))+\sum_{a=1}^{p-1} \bar{\chi}(1-\bar{a})\right) \\
& =-\tau(\chi)\left(-2+\sum_{a=1}^{p-1} \bar{\chi}(a(1-a))\right)
\end{aligned}
$$

Now note that $|\tau(\chi)|=\sqrt{p}$, if $\chi \neq \chi_{0}$. From (2.1) and (2.2) we may immediately deduce Lemma 1.

Lemma 2 Let $p$ be an odd prime, $\chi$ be any non-real character $\bmod p$. Then we have the identity

$$
\sum_{a=1}^{p-1} \chi(a(a-1))=\frac{\tau^{2}(\chi)}{\tau\left(\chi^{2}\right)}
$$

Therefore,

$$
\left|\sum_{a=1}^{p-1} \chi(a(a-1))\right|=\sqrt{p}
$$

Proof From the definition and properties of the classical Gauss sums we have

$$
\begin{aligned}
\tau^{2}(\chi) & =\sum_{a=1}^{p-1} \sum_{b=1}^{p-1} \chi(a) \chi(b) e\left(\frac{a+b}{p}\right)=\sum_{a=1}^{p-1} \chi(a) \sum_{b=1}^{p-1} \chi^{2}(b) e\left(\frac{b(a+1)}{p}\right) \\
& =\tau\left(\chi^{2}\right) \sum_{a=1}^{p-1} \chi(a) \bar{\chi}\left((a+1)^{2}\right)=\tau\left(\chi^{2}\right) \sum_{a=2}^{p} \chi(a-1) \bar{\chi}\left(a^{2}\right) \\
& =\tau\left(\chi^{2}\right) \sum_{a=2}^{p-1} \chi((1-\bar{a}) \bar{a})=\tau\left(\chi^{2}\right) \sum_{a=1}^{p-1} \chi(a(1-a))
\end{aligned}
$$

or

$$
\sum_{a=1}^{p-1} \chi(a(a-1))=\frac{\tau^{2}(\chi)}{\tau\left(\chi^{2}\right)}
$$

This proves Lemma 2.

\section{Proof of the theorem}

In this section, we shall complete the proof of our theorem. First from the orthogonality of characters $\bmod p$ we have

$$
\left.\left.\sum_{\chi \bmod p}\left|\sum_{m=1}^{p-1} \chi(m)\right| \sum_{a=1}^{p-1} e\left(\frac{m a^{3}+n a}{p}\right)\right|^{2}\right|^{2}=(p-1) \sum_{m=1}^{p-1}\left|\sum_{a=1}^{p-1} e\left(\frac{m a^{3}+n a}{p}\right)\right|^{4} .
$$


On the other hand, if $3 \nmid p-1$, then any non-principal character $\chi$ is not a 3 -order character $\bmod p$. Note that

$$
\begin{aligned}
& \sum_{m=1}^{p-1} \chi_{0}(m)\left|\sum_{a=1}^{p-1} e\left(\frac{m a^{3}+n a}{p}\right)\right|^{2} \\
& =\sum_{a=1}^{p-1} \sum_{b=1}^{p-1} \sum_{m=1}^{p-1} e\left(\frac{m b^{3}\left(a^{3}-1\right)+n b(a-1)}{p}\right) \\
& =(p-1)^{2}+p-2=p^{2}-p-1 .
\end{aligned}
$$

From (3.2) and Lemma 1 we have

$$
\begin{aligned}
& \left.\left.\sum_{\chi \bmod p}\left|\sum_{m=1}^{p-1} \chi(m)\right| \sum_{a=1}^{p-1} e\left(\frac{m a^{3}+n a}{p}\right)\right|^{2}\right|^{2} \\
& =\left.\left.\left|\sum_{m=1}^{p-1} \chi_{0}(m)\right| \sum_{a=1}^{p-1} e\left(\frac{m a^{3}+n a}{p}\right)\right|^{2}\right|^{2}+\left.\left.\sum_{\substack{\chi \bmod \\
\chi \neq \chi_{0}}}\left|\sum_{m=1}^{p-1} \chi(m)\right| \sum_{a=1}^{p-1} e\left(\frac{m a^{3}+n a}{p}\right)\right|^{2}\right|^{2} \\
& =\left(p^{2}-p-1\right)^{2}+\sum_{\substack{\chi \bmod p \\
\chi \neq \chi_{0}}} p^{2} \cdot\left|\sum_{a=1}^{p-2} \bar{\chi}\left(3 a^{2}+3 a+1\right)\right|^{2} \\
& =\left(p^{2}-p-1\right)^{2}+p^{2} \sum_{\chi \bmod p}\left|\sum_{a=1}^{p-2} \bar{\chi}\left(3 a^{2}+3 a+1\right)\right|^{2} \\
& -p^{2}\left(\sum_{a=1}^{p-2} \chi_{0}\left(3 a^{2}+3 a+1\right)\right)^{2} \\
& =\left(p^{2}-p-1\right)^{2}+p^{2}(p-1) \sum_{\substack{a=1 \\
a^{2}+a \equiv b^{2}+b \bmod p}}^{p-2} \sum_{\substack{p-2 \\
p-2}}^{2} 1-p^{2}(p-2)^{2} \\
& =\left(p^{2}-p-1\right)^{2}+p^{2}(p-1) \quad \sum_{\substack{a=1 \\
(a-b)(a+b+1) \equiv 0 \bmod p}}^{p-2} \sum_{b=1}^{p-2} 1-p^{2}(p-2)^{2} \\
& =\left(p^{2}-p-1\right)^{2}+p^{2}(p-1)(p-2+p-2-1)-p^{2}(p-2)^{2} \\
& =(p-1)\left(2 p^{3}-3 p^{2}-3 p-1\right) \text {. }
\end{aligned}
$$

If $3 \nmid p-1$, then combining (3.1) and (3.3) we may immediately deduce the identity

$$
\sum_{m=1}^{p-1}\left|\sum_{a=1}^{p-1} e\left(\frac{m a^{3}+n a}{p}\right)\right|^{4}=2 p^{3}-3 p^{2}-3 p-1
$$

or

$$
\sum_{m=1}^{p}\left|\sum_{a=1}^{p-1} e\left(\frac{m a^{3}+n a}{p}\right)\right|^{4}=2 p^{3}-3 p^{2}-3 p .
$$


If $3 \mid p-1$, let $\chi_{1} \neq \chi_{0}$ be a 3 -order character $\bmod p$, then $\bar{\chi}_{1}=\chi_{1}^{2}$ is also a 3 -order character $\bmod p$; this time note that

$$
\sum_{m=1}^{p-1} \chi_{0}(m)\left|\sum_{a=1}^{p-1} e\left(\frac{m a^{3}+n a}{p}\right)\right|^{2}=p(p-1)-2 p-1=p^{2}-3 p-1
$$

and

$$
\begin{aligned}
\sum_{a=1}^{p-2} \chi_{1}\left((a+1)^{3}-a^{3}\right) & =\sum_{a=1}^{p-2} \chi_{1}\left((\bar{a}+1)^{3}-1\right)=\sum_{a=1}^{p-1} \chi_{1}\left(a^{3}-1\right) \\
& =\sum_{a=1}^{p-1}\left(\bar{\chi}_{1}^{2}(a)+\bar{\chi}_{1}(a)+1\right) \chi_{1}(a-1) \\
& =-2+\sum_{a=1}^{p-1} \chi_{1}(a(1-a)),
\end{aligned}
$$

and from Lemma 2 and the method of proving (3.3) we have

$$
\begin{aligned}
\left.\left.\sum_{\chi \bmod p}\left|\sum_{m=1}^{p-1} \chi(m)\right| \sum_{a=1}^{p-1} e\left(\frac{m a^{3}+n a}{p}\right)\right|^{2}\right|^{2} & \\
= & \left(p^{2}-3 p-1\right)^{2}+\sum_{\substack{\chi \bmod p \\
\chi \neq \chi_{0}, \chi_{1}, \chi_{1}^{2}}} p^{2}\left|\sum_{a=1}^{p-2} \bar{\chi}\left(3 a^{2}+3 a+1\right)\right|^{2} \\
& +2 p\left|-2+\sum_{a=1}^{p-1} \chi_{1}(a(1-a))\right|^{2} \\
= & \left(p^{2}-3 p-1\right)^{2}+p^{2}(p-1)(2 p-5)-2 p^{2}\left|\sum_{a=1}^{p-2} \chi_{1}\left((a+1)^{3}-a^{3}\right)\right|^{2} \\
& +2 p\left(p+4-2 \sum_{a=1}^{p-1} \chi_{1}(a(1-a))-2 \sum_{a=1}^{p-1} \bar{\chi}_{1}(a(1-a))\right)-p^{2}(p-4)^{2} \\
= & \left(p^{2}-3 p-1\right)^{2}+p^{2}(p-1)(2 p-5)-p^{2}(p-4)^{2} \\
& -\left(2 p^{2}-2 p\right)\left(p+4-2 \sum_{a=1}^{p-1} \chi_{1}(a(1-a))-2 \sum_{a=1}^{p-1} \bar{\chi}_{1}(a(1-a))\right) \\
= & (p-1)\left(2 p^{3}-5 p^{2}-15 p-1\right)+4(p-1)\left(\tau^{3}\left(\chi_{1}\right)+\tau^{3}\left(\bar{\chi}_{1}\right)\right) .
\end{aligned}
$$

So if $3 \mid p-1$, then combining (3.1) and (3.5) we can deduce the asymptotic formula

$$
\begin{aligned}
\sum_{m=1}^{p}\left|\sum_{a=1}^{p-1} e\left(\frac{m a^{3}+n a}{p}\right)\right|^{4} & =1+\sum_{m=1}^{p-1}\left|\sum_{a=1}^{p-1} e\left(\frac{m a^{3}+n a}{p}\right)\right|^{4} \\
& =2 p^{3}-5 p^{2}-15 p+4\left(\tau^{3}\left(\chi_{1}\right)+\tau^{3}\left(\bar{\chi}_{1}\right)\right) .
\end{aligned}
$$

Now our theorem follows from (3.4) and (3.6). 
Competing interests

The authors declare that they have no competing interests.

\section{Authors' contributions}

The main idea of this paper was proposed by $\mathrm{MZ}$ and DH. All authors contributed equally to the writing of this paper. All authors read and approved the final manuscript.

\section{Author details}

${ }^{1}$ School of Science, Xi'an Polytechnic University, Xi'an, Shaanxi, P.R. China. ${ }^{2}$ Department of Mathematics, Northwest University, Xi'an, Shaanxi, P.R. China.

\section{Acknowledgements}

The authors would like to thank the referee for his very helpful and detailed comments, which have significantly improved the presentation of this paper. This work is supported by the P.S.F. (2013JZ001) and N.S.F. (11371291) of P.R. China, and the SF of Education of Shaanxi province (12JK0877).

Received: 7 February 2014 Accepted: 6 March 2014 Published: 19 March 2014

References

1. Cochrane, T, Zheng, Z: Pure and mixed exponential sums. Acta Arith. 91, 249-278 (1999)

2. Cochrane, T, Zheng, Z: Bounds for certain exponential sums. Asian J. Math. 4, 757-774 (2000)

3. Cochrane, T, Zheng, Z: Upper bounds on a two-term exponential sums. Sci. China Ser. A 44, 1003-1015 (2001)

4. Cochrane, T, Pinner, C: A further refinement of Mordell's bound on exponential sums. Acta Arith. 116, 35-41 (2005)

5. Cochrane, T, Pinner, C: Using Stepanov's method for exponential sums involving rational functions. J. Number Theory 116, 270-292 (2006)

6. Apostol, TM: Introduction to Analytic Number Theory. Springer, New York (1976)

doi:10.1186/1029-242X-2014-116

Cite this article as: Zhu and Han: Retracted: On the fourth power mean of the two-term exponential sums. Journal of Inequalities and Applications 2014 2014:116.

\section{Submit your manuscript to a SpringerOpen ${ }^{\circ}$ journal and benefit from:}

- Convenient online submission

- Rigorous peer review

- Immediate publication on acceptance

- Open access: articles freely available online

- High visibility within the field

- Retaining the copyright to your article 